\title{
Prostate cancer screening: going beyond the clinical evidence
}

\author{
Murray Krahn MD MSc
}

See related guideline on page 1225 and at www.cmaj.ca/lookup/doi/10.1503/cmaj.140703

I $\mathrm{n}$ a linked guideline, the Canadian Task Force on Preventive Health Care summarizes its updated recommendations not to use the prostate-specific antigen (PSA) test for prostate cancer screening. ${ }^{1}$ The task force's guideline is an excellent example of health care decisions being made from the perspective of evidence-based medicine. The task force focused on clinically meaningful outcomes and rated the quality of evidence and strength of recommendations using the Grading of Recommendations Assessment, Development and Evaluation (GRADE; gradeworkinggroup.org) system. However, it paid insufficient attention to patient values, patient preferences and costs.

A story: In the mid-1990s, I participated in a PSA guideline group for Cancer Care Ontario. The "usual" literature search was conducted, which focused on the evidence of mortality benefit and the potential harms of screening. I had been building decision models of PSA screening ${ }^{2}$ and measuring cost and quality-of-life outcomes for years. I was slightly chagrined that none of my papers turned up in the search. It made me ask, "Why are the rest of the committee and I seeing this problem so differently?" My model suggested that there might be a mortality benefit but overall a loss of quality-adjusted life expectancy. This meant that the way in which patients valued health outcomes was a key part of the screening decision. In other words, this was a "preference-sensitive" decision. Moreover, it seemed too obvious to mention that cost was a relevant concern in a publicly funded health system. The rest of the committee, though, did not see evidence on quality of life, patient preferences or cost as relevant. They also did not see modelling as a serious scientific endeavour that might illumine the key trade-offs (mortality v. quality of life). They saw the problem as one of whether PSA screening reduced mortality.

Flash forward roughly 20 years. Plus ça change ... We have more data but the same dilemma. Trials of PSA screening have been reported and show a possible but small mortality benefit. The adverse effects of prostate cancer treatment are known. Modelling studies predict a small mortality gain and a questionable overall health benefit. ${ }^{3,4}$ There is more evidence about $\cos ^{5}$ and patient preferences. ${ }^{6,7}$ Yet guideline panels such as the US Preventive Services Task Force and the Canadian Task Force on Preventive Health Care are still basing their recommendations only on the trial evidence.

I have come to believe that there are at least three paradigms at work in the interpretation of scientific evidence: evidence-based medicine; health economics and decision science; and social science and bioethics. Researchers who work mainly within one of these paradigms ask slightly different questions. What inferences can be drawn from the evidence? What is the best decision given the evidence we have? What decision fits with patient and social values? Each paradigm privileges different things: clinical outcomes; outcomes, costs and preferences; patient experiences, power relationships and ethical principles. Each is a lens that subtly conditions what we consider to be evidence, how we integrate evidence and how we recommend a course of action.

If the Canadian task force's guideline panel had been composed solely of sociologists, psychologists and ethicists, my bet is that we would have heard more about patient choice and empowerment. If the panel had comprised health economists and decision analysts, we would have heard about preference-sensitive decisions and cost-effectiveness.

As it stands, the task force's guideline provides a good summary of the data on the effectiveness of prostate cancer screening and a reasonable review

\section{KEY POINTS}

- Recommendations for clinical practice should be based not only on evidence of outcomes, but also on patient preferences, social values and costs to the health care system.

- Patient preferences, particularly for preference-sensitive decisions, require attention, formal study and weight in clinical and policy decision-making.

- Whether to screen for prostate cancer is a preference-sensitive decision. 
of the rate at which potential harms occur. However, the report does not include a comprehensive review of patient harms; yes, they happen, but are they important? There is no review of modelling studies. Several international groups are modelling prostate cancer screening ${ }^{3,4}$ and, in my view, offer the best extant look at the balance of potential harms and benefits of screening. The guideline gives only a brief nod to patient preference and shared decision-making. An enormous amount has been published on this topic. ${ }^{8}$ As of 2008, for example, there were 18 published trials of shared decision-making in prostate cancer. ${ }^{9}$ Internationally, there is a growing literature on the integration of patient values and preferences into clinical practice guidelines, and on patient-based health technology assessments. ${ }^{10,11}$ Finally, the guideline contains no review of the evidence on cost, because unfortunately it is outside the task force's purview.

Other published guidelines open the door slightly wider to patient choice. ${ }^{12}$ There clearly is not enough evidence to mount an organized screening program. However, the falling overall mortality in some countries that screen intensively, the evidence that treatment may have a very modest disease-specific mortality benefit, and the highly variable preferences for treatment outcomes suggest to me that we should not push patients out of decision-making in this area.

\section{References}

1. Canadian Task Force on Preventive Health Care. Recommendations on screening for prostate cancer with the prostate-specific antigen test. CMAJ 2014; 186:1225-34.

2. Krahn MD, Mahoney JE, Eckman MH, et al. Screening for prostate cancer. A decision analytic view. JAMA 1994;272:773-80.

3. Heijnsdijk EAM, Wever EM, Auvinen A, et al. Quality-of-life effects of prostate-specific antigen screening. N Engl J Med 2012; 367:595-605.

4. Pataky R, Gulati R, Etzioni R, et al. Is prostate cancer screening cost-effective? A microsimulation model of prostate-specific antigen-based screening for British Columbia, Canada. Int $J$ Cancer 2014;135:939-47.

5. Krahn MD, Zagorski B, Laporte A, et al. Healthcare costs associated with prostate cancer: estimates from a population-based study. BJU Int 2010;105:338-46.

6. Krahn M, Ritvo P, Naglie G, et al. Construction of the PatientOriented Prostate Utility Scale (PORPUS): a multiattribute health state classification system for prostate cancer. $J$ Clin Epidemiol 2000;53:920-30.

7. Krahn M, Ritvo P, Irvine J, et al. Patient and community preferences for outcomes in prostate cancer: implications for clinical policy. Med Care 2003;41:153-64.

8. Knight SJ. Decision making and prostate cancer screening. Urol Clin North Am 2014;41:257-66.

9. Krahn M, Naglie G. The next step in guideline development: incorporating patient preferences. JAMA 2008:300:436-8.

10. Boivin A, Green J, van der Meulen J, et al. Why consider patients' preferences? A discourse analysis of clinical practice guideline developers. Med Care 2009;47:908-15.

11. Krahn M. "New" evidence for clinical practice guidelines. Patient 2010;3:71-7.

12. Qaseem A, Barry MJ, Denberg TD, et al. Screening for prostate cancer: a guidance statement from the Clinical Guidelines Committee of the American College of Physicians. Ann Intern Med 2013;158:761-9.

Affiliations: Toronto Health Economics and Technology Assessment Collaborative; Department of Medicine and Faculty of Pharmacy, University of Toronto and University Health Network, Toronto, Ont.

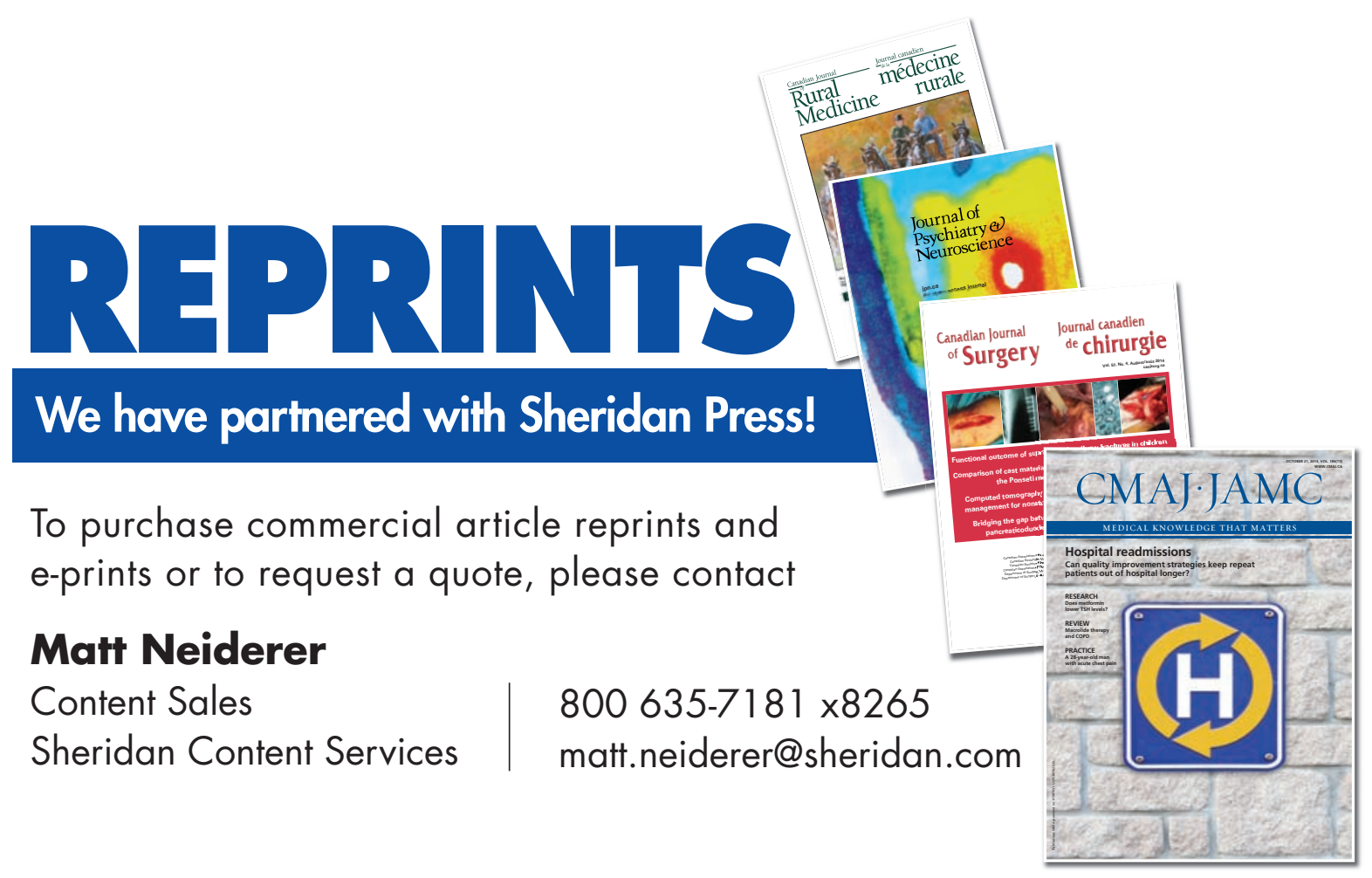

